\title{
Case Report: Prune perineum syndrome: A rare case with an
}

\section{unfavourable outcome [version 1; peer review: 1 approved, 2}

\section{approved with reservations]}

\author{
Roberto I. Lopes (Di)1,2, Francisco T. Dénes¹, Gustavo B. Messi', \\ Marcos G. Machado1 \\ ${ }^{1}$ Division of Urology, University of São Paulo Medical School, São Paulo, Brazil \\ ${ }^{2}$ Division of Urology, The Hospital for Sick Children, Toronto, Canada
}

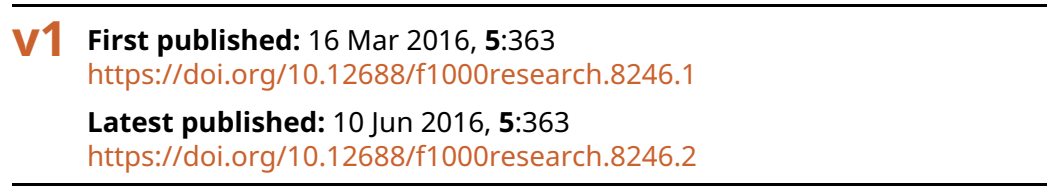

\section{Abstract}

Prune perineum syndrome (PPS) is a rare anomaly, with only two previous case reports, both dying in the perinatal period. We report the first case of PPS that reached childhood.

The patient presented with hypoplastic genitalia and bilateral cryptorchidism. There was no evidence of an anal orifice. A significant prune-like mass was observed, extending from the perineum to both gluteal regions and to a cephalic mid-line bony prominence, with a $1 \mathrm{~cm}$ central orifice that discharged urine. MRI confirmed the previous findings and revealed a right crossed ectopic kidney, intestinal malrotation, a hypoplastic infrarenal inferior vena cava and a hypoplastic right iliac artery. Endoscopic evaluation through the orifice revealed a cavity lined by urothelial mucosa, with a small communication to the anterior urethra in its anterior wall.

A staged reconstruction was planned, with a first-step urinary diversion through a continent abdominal reservoir associated to bilateral orchiopexy. The patient was discharged from the hospital three weeks later under intermittent catheterization. The next planned surgical step was the resection of the perineal mass and its cavity associated to the removal of the prominent sacrococcygeal bones. Unfortunately, four months after the first surgery the patient developed an acute abdomen and underwent a laparotomy that revealed a necrotic ileal segment secondary to obstructive adherences. He developed severe malabsorption followed by septic shock, dying five weeks after the procedure.

Due to the lack of literature, there is no consensus for the management of these cases. The wish of the family for a better quality of life and social acceptance, compelled us to perform a urinary diversion, to be followed by a plastic and orthopedic reconstruction. Despite the successful initial result, the patient developed a late abdominal obstruction that was misdiagnosed, precipitating his

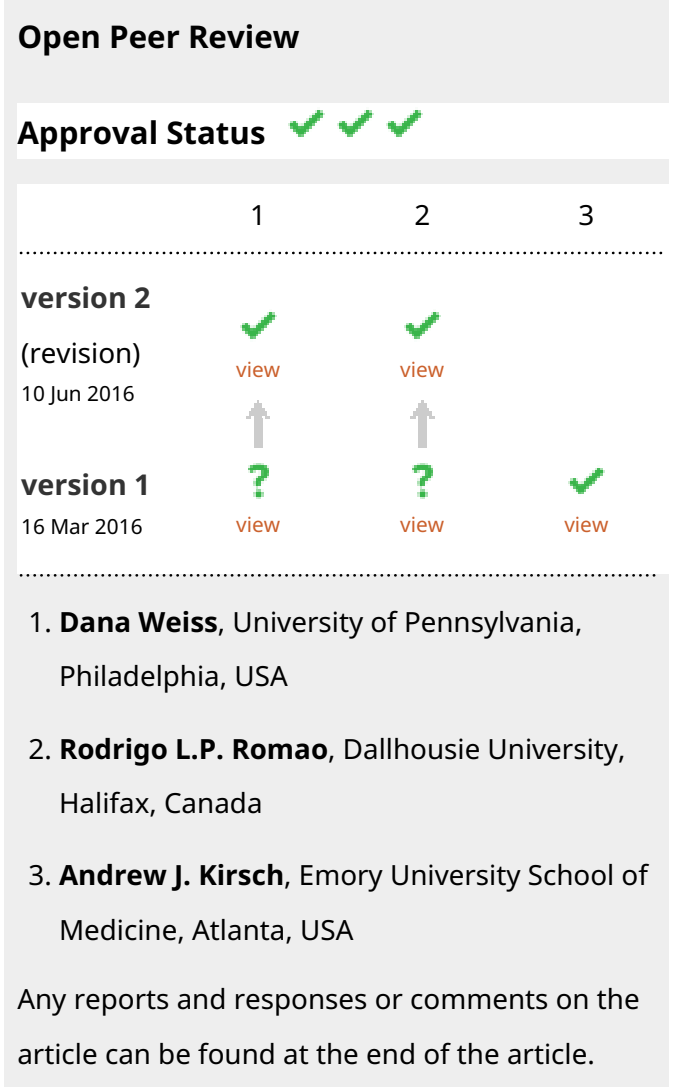


untimely death five months after the first procedure.

\section{Keywords}

Prune perineum syndrome, pelvic malformations, congenital disease

, urinary tract

Corresponding author: Roberto I. Lopes (roberto.iglesiaslopes@sickkids.ca)

Competing interests: No competing interests were disclosed.

Grant information: The author(s) declared that no grants were involved in supporting this work.

Copyright: $\odot 2016$ Lopes RI et al. This is an open access article distributed under the terms of the Creative Commons Attribution License, which permits unrestricted use, distribution, and reproduction in any medium, provided the original work is properly cited.

How to cite this article: Lopes RI, Dénes FT, Messi GB and Machado MG. Case Report: Prune perineum syndrome: A rare case with an unfavourable outcome [version 1; peer review: 1 approved, 2 approved with reservations] F1000Research 2016, 5:363 https://doi.org/10.12688/f1000research.8246.1

First published: 16 Mar 2016, 5:363 https://doi.org/10.12688/f1000research.8246.1 


\section{Introduction}

Prune perineum was first described in 1979 by Peeden et al. ${ }^{1}$ and to our knowledge only one further case has been presented ${ }^{2}$. PPS includes significant abnormalities in the development of the caudal axis, with characteristic deformation of the sacrococcygeal spine associated with a large wrinkled perineal mass and a hypoplastic external genitalia. In both previously described cases, the patient died in the perinatal period ${ }^{1,2}$. We present the first case of a male born with this disease who reached 5 years of age.

\section{Case report}

The patient was born at the $39^{\text {th }}$ week of the uneventful first gestation of a 28-year-old woman, without any family history of congenital malformation or chronic illness. The maternal medical history was uneventful and pregnancy was normal until the last trimester, when control ultrasonography disclosed a fetal perineal abnormality, therefore a cesarean delivery was performed. A large perineal mass, associated to imperforated anus was readily identified. Urine was observed leaking through an orifice in the perineal mass. A left flank terminal colostomy was performed on the second day of life. Post-operatively, the patient presented with good clinical course, with functioning colostomy and preserved renal function, being discharged after 2 weeks. At four years of age, he came to our service without any previous significant clinical problems, including urinary tract infection. The somatic growth and neuro-psycho-motor development were normal. He had no medication, was wearing diapers and a colostomy bag. Despite a small limp, he was able to walk normally.

At physical examination, all abnormalities were below the umbilicus. The abdomen had a functioning left colostomy while the genital area presented hypoplastic male external genitalia with bilateral impalpable cryptorchidism. There was no evidence of an anal orifice. The right leg was shorter than the left (Figure 1). Posteriorly, a $20 \times 15 \times 8 \mathrm{~cm}$ prune-like mass was observed, extending from the perineum to both gluteal regions and to a cephalic mid-line bony prominence, with a $1 \mathrm{~cm}$ central orifice that discharged urine. The corrugated skin of the prune-like mass could be easily lifted, disclosing, through its orifice, a large cavity below that contained urine (Figure 2). In the sitting position, the mass and its cavity were compressed against the perineum.

Laboratory examination showed a normal blood count and renal function, and the karyotype was 46, XY. Pelvic radiographs demonstrated an exaggerated sacrococcygeal concavity, lumbar dysrrhaphism and pubic dyastasis. The dorsal bony prominence corresponded to the sacrococcygeal spine, whose abnormal concavity oriented its tip upward, like a small tail. The contrast injection through the orifice of the mass showed a large cavity that extended sagittally and laterally. MRI confirmed the previous findings and revealed a right crossed ectopic kidney, intestinal malrotation, a hypoplastic infrarenal inferior vena cava and a hypoplastic right iliac artery. The posterior concavity of sacrococcygeal bones, associated to a deformity of the pelvic rim was evident, as well as the cavity below the convoluted skin and subcutaneous layer of the perineal mass (Figure 3). The endoscopic evaluation through the orifice revealed a cavity lined by urothelial mucosa, with a small communication to the anterior urethra in its anterior wall. The

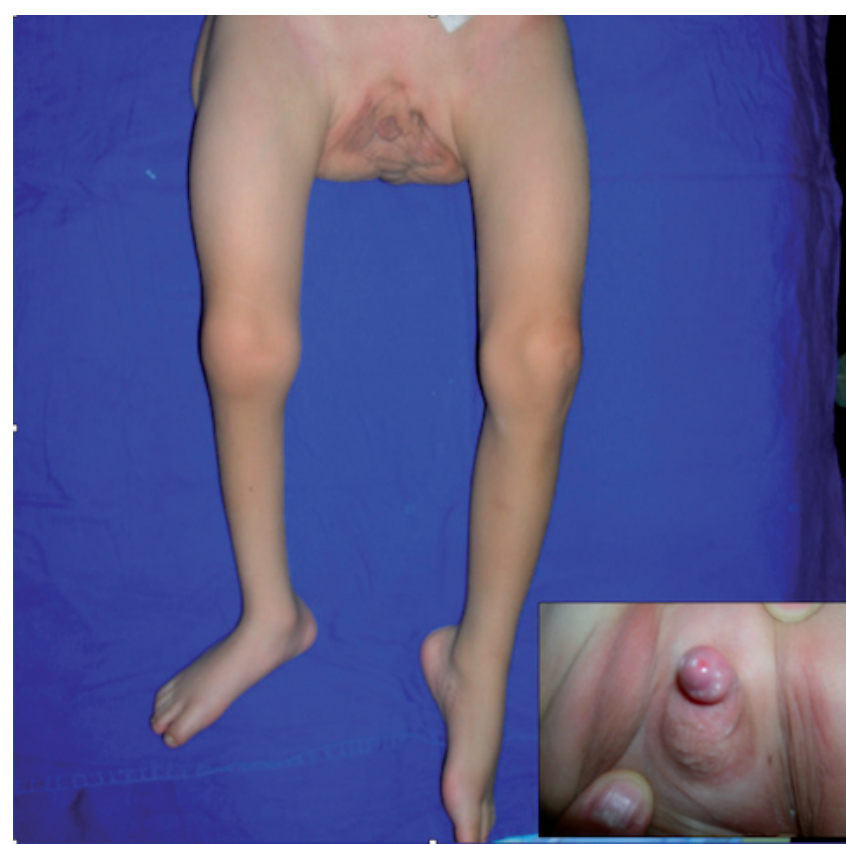

Figure 1. Anterior view of perineum and lower limbs. Hypoplastic external genitals in detail.

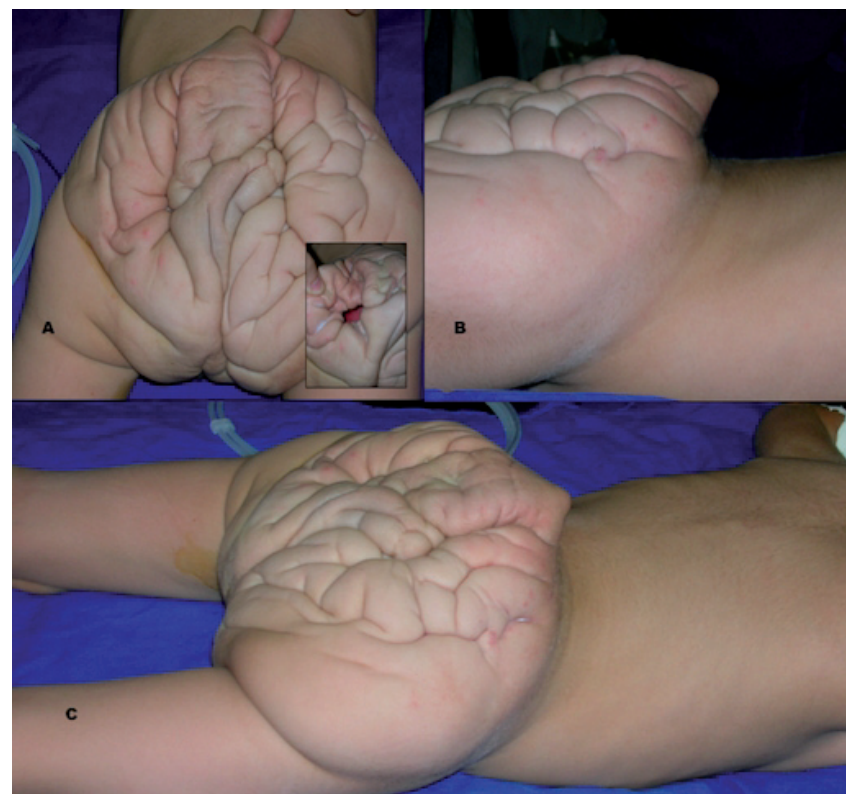

Figure 2. Posterior view showing perineal mass with central orifice in detail (A). Lateral view (B), and perspective (C).

ureteral orifices were not identified, although urine accumulated in the cavity during the examination.

After extensive evaluation of the case and considering the psychological effects of the malformation, as well as the parental wish for a better quality of life and social acceptance of the child, 


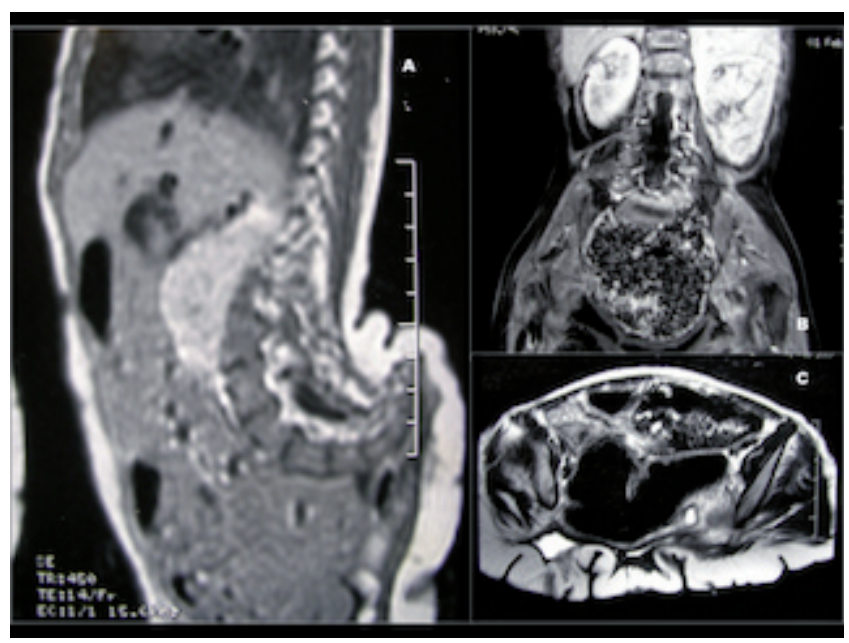

Figure 3. Nuclear magnetic resonance demonstrating exaggerated sacrococcygeal convexity (A); crossing ectopic right kidney and an intestinal malrotation (B); absence of pubic bone, and the corrugated skin covering the posterior perineal cavity $(\mathbf{C})$.

we planned a staged reconstruction, with a first-step urinary diversion through a continent abdominal reservoir associated to bilateral orchiopexy. The procedure was successful and the patient recovered uneventfully. He was discharged from the hospital three weeks later with a dry perineal cavity and under intermittent catheterization regularly performed by the mother. The next surgical step would be the resection of the perineal mass and its cavity associated to the removal of the prominent sacrococcygeal bones. Unfortunately, four months after the first surgery the patient developed an acute abdomen that was misdiagnosed and treated as a urinary tract infection elsewhere. Forty-eight hours later, he was transferred to our service and was submitted to exploratory laparotomy that revealed a necrotic ileal segment secondary to obstructive adherences. The resection of the necrotic segment associated to a terminal ileostomy was performed, but he developed severe malabsorption followed by septic shock, dying five weeks after the procedure.

\section{Discussion}

Previously, only two cases of the rare prune perineum syndrome have been described, and both patients died in the perinatal period due to sepsis ${ }^{1,2}$. Early colostomy and normal renal function by an unobstructed solitary kidney enabled long-term survival in our case. Contrary to the two previous cases, this was a male patient with a $46 \mathrm{XY}$ karyotype, although with a very small penis and impalpable testes.

Due to the lack of literature, there is no consensus for the management of these unfortunate children. We report the unsuccessful outcome of a child treated for this syndrome. Even though he presented a quite stable physical development without any neurological impairment up to the age of four years, the wish of the family for a better quality of life and social acceptance, compelled us to perform a urinary diversion, to be followed by a plastic and orthopedic reconstruction. Despite the successful initial result, the patient developed a late abdominal obstruction that was misdiagnosed, precipitating his untimely death five months after the first procedure.

\section{Consent}

Written informed consent was obtained from the parent of the patient for publication of this case report and any accompanying images and/or other details that could potentially reveal the patient's identity.

\section{Author contributions}

RIL and GBM retrieved patient data, reviewed the literature and wrote the manuscript. MGM and FTD did a critical revision of the manuscript for scientific and factual content. All authors approved the final version for publication.

\section{Competing interests}

No competing interests were disclosed.

\section{Grant information}

The author(s) declared that no grants were involved in supporting this work.
1. Peeden JN Jr, Wilroy RS Jr, Soper RG: Prune perineum. Teratology. 1979; 20(2): 233-236.

PubMed Abstract | Publisher Full Text
2. Williams DA, Weiss $\mathrm{T}$, Wade $\mathrm{E}$, et al.: Prune perineum syndrome: report of a second case. Teratology. 1983; 28(1): 145-148. PubMed Abstract | Publisher Full Text 


\title{
Open Peer Review
}

\section{Current Peer Review Status:}

\section{Version 1}

Reviewer Report 31 May 2016

https://doi.org/10.5256/f1000research.8868.r14064

(C) 2016 Kirsch A. This is an open access peer review report distributed under the terms of the Creative Commons Attribution License, which permits unrestricted use, distribution, and reproduction in any medium, provided the original work is properly cited.

\begin{abstract}
Andrew J. Kirsch
Department of Pediatric Urology, Children's Healthcare of Atlanta, Emory University School of Medicine, Atlanta, GA, USA

The authors present a fascinating case of Prune Perineum Syndrome - the first case of survival beyond infancy. Only two others have been reported with PPS over the past half century. The experienced group of pediatric surgeons describe this exceedingly rare condition and offer the child an opportunity to improve quality of life through surgical reconstruction. The preoperative imaging studies are highly educational and instructive. Unfortunately, a delayed postoperative complication and poor access to emergency medical care lead to the patient's demise. This report, however, provides important clinical information to the limited available literature on this condition. This case report will help to educate and guide future generations of patients and their families, as well as physicians and surgeons in the diagnosis and management of PPS.
\end{abstract}

Competing Interests: No competing interests were disclosed.

I confirm that I have read this submission and believe that I have an appropriate level of expertise to confirm that it is of an acceptable scientific standard.

Reviewer Report 20 May 2016

https://doi.org/10.5256/f1000research.8868.r12915

(C) 2016 Romao R. This is an open access peer review report distributed under the terms of the Creative Commons Attribution License, which permits unrestricted use, distribution, and reproduction in any medium, provided the original work is properly cited.

\section{Rodrigo L.P. Romao}

Division of Urology, Division of Pediatric General Surgery, Department of Surgery, IWK Health 
Centre, Dallhousie University, Halifax, NS, Canada

The authors report a case of prune-perineum, an extremely rare malformation for which survival beyond the perinatal period has not been documented. Only 2 previous case reports are described in the literature.

The description of the findings is interesting and the illustrations are of good quality. The description of the surgical technique is short. If the authors were able to provide more details about the reconstruction performed, it would be of interest to the reader. It is not clear what kind of reservoir was created and how exactly CIC was conducted postoperatively. A detailed account of the findings and intra-operative decision-making process would strengthen the report significantly.

The authors should be applauded for reporting the best outcome thus far for this exceedingly rare and complex condition.

Competing Interests: No competing interests were disclosed.

\section{I confirm that I have read this submission and believe that I have an appropriate level of expertise to confirm that it is of an acceptable scientific standard, however I have significant reservations, as outlined above.}

Author Response 31 May 2016

Roberto Iglesias Lopes, University of São Paulo Medical School, São Paulo, Brazil

If the authors were able to provide more details about the reconstruction performed, it would be of interest to the reader. It is not clear what kind of reservoir was created and how exactly CIC was conducted postoperatively. A detailed account of the findings and intraoperative decision-making process would strengthen the report significantly.

Answer: A perineal orifice communicated to a cavity, where we identified a bladder, which was abnormally located perineally. The orifice was at least $1 \mathrm{~cm}$ large, and all urine leaked through this orifice. Nevertheless, on cystoscopic evaluation, a guidewire was inserted through the urethra and reached the bladder (the urethra was patent, but very thin). A patent, albeit very thin urethra was connected between the perineal cavity (bladder) and the hypoplastic penile shaft. The bladder neck was also very small. As the bladder consisted of a cavity lined with urothelium and was not a true bladder, we opted for a continent bowel reservoir. Only one ureter was implanted to it, as the patient had only one kidney. A true bladder was not available and therefore, a continent cutaneous ileal neobladder was performed, with the appendix used for clean intermittent catheterization every four hours in the postoperative care.

Competing Interests: No competing interests were disclosed. 


\section{https://doi.org/10.5256/f1000research.8868.r13606}

(c) 2016 Weiss D. This is an open access peer review report distributed under the terms of the Creative Commons Attribution License, which permits unrestricted use, distribution, and reproduction in any medium, provided the original work is properly cited.

\section{Dana Weiss}

Division of Urology, Departments of Surgery, The Children's Hospital of Philadelphia, Perelman School of Medicine, University of Pennsylvania, Philadelphia, PA, USA

The authors present a very interesting and unique case report.

Some questions that arise:

Had the child had any renal imaging after birth? Was the urine leaking through a penile urethra as well as through the orifice that contained urine? This part is not clear from the description.

The authors could assist the reader additionally, should this case ever arise again, by describing exactly what kind of reconstruction was done at the first stage. Was a true bladder found? Were the ureters implanted normally into a bladder? Was there a bladder neck and urethra? What exactly was the "orifice" that was draining urine? When the authors say they created a continent abdominal reservoir - was this a bowel reservoir to which the ureters were implanted? If there was any bladder present, was this used?

This kind of case report is interesting and important since many pediatric surgeons and urologists may never see something like this in our lifetime, but if we do, it is very valuable to have some inkling of what is going on, and how we might even begin to manage it.

Competing Interests: No competing interests were disclosed.

\section{I confirm that I have read this submission and believe that I have an appropriate level of expertise to confirm that it is of an acceptable scientific standard, however I have significant reservations, as outlined above.}

Author Response 11 May 2016

Roberto Iglesias Lopes, University of São Paulo Medical School, São Paulo, Brazil

\section{Author Response to Reviewer's Comments:}

Had the child had any renal imaging after birth?

Answer: He was referred to us at age 4, having undergone previously a colostomy elsewhere on the second day of life. We started all diagnostic workup from point zero.

Was the urine leaking through a penile urethra as well as through the orifice that contained urine?

Answer: The perineal orifice communicated to a cavity, where we identified a bladder, which was abnormally located perineally. The orifice was at least $1 \mathrm{~cm}$ large, and all urine leaked through this orifice. Nevertheless, on cystoscopic evaluation, a guidewire was inserted 
through the urethra and reached the bladder (the urethra was patent, but very thin).

Was a true bladder found?

Answer: Besides the large perineal cavity (identified as bladder) drained by the orifice and connected to the urethra, there was no other structure resembling a normal bladder.

Were the ureters implanted normally into a bladder?

Answer: As urine was continuously flowing into the perineal cavity (bladder) and leaking through the perineal cavity, it was evident that the ureter (draining alone the right crossed ectopic kidney) reached the perineal cavity. Nevertheless, on "cystoscopic" evaluation we weren't able to find the ureteral orifice.

Was there a bladder neck and urethra?

Answer: As mentioned before, a patent, albeit very thin urethra was connected between the perineal cavity (bladder) and the hypoplastic penile shaft. The bladder neck was also very small.

What exactly was the "orifice" that was draining urine?

Answer: We do not have an embryologic explanation for it, and we can only postulate that a fistula eventually ruptured between the "perineal bladder" and skin before birth due to increased intravesical pressure (the perineal wrinkled skin was very thin and had no bony support).

When the authors say they created a continent abdominal reservoir - was this a bowel reservoir to which the ureters were implanted?

Answer: Exactly: it was a continent bowel reservoir, but only one ureter was implanted to it, as the patient had only one kidney.

If there was any bladder present, was this used?

Answer: The presumptive primitive bladder, namely the perineal cavity, was left in situ, for posterior removal. It became completely dry after surgery.

Competing Interests: None 
The benefits of publishing with F1000Research:

- Your article is published within days, with no editorial bias

- You can publish traditional articles, null/negative results, case reports, data notes and more

- The peer review process is transparent and collaborative

- Your article is indexed in PubMed after passing peer review

- Dedicated customer support at every stage

For pre-submission enquiries, contact research@f1000.com 